\title{
PECULIARITIES OF ELECTRON TRANSPORT IN PbTe:Cr DUE TO PRESENCE OF RESONANT IMPURITY STATE*
}

\author{
E. Grodzicka, W. Dobrowolski, J. Kossut, T. Story \\ AND B. WITKOWSKA \\ Institute of Physics, Polish Academy of Sciences \\ Al. Lotników 32/46, 02-668 Warsaw, Poland
}

The results of transport investigation of $\mathrm{Pb}_{1-x} \mathrm{Cr}_{x} \mathrm{Te}(x \leq 0.009)$ in temperature range $3.5-300 \mathrm{~K}$ are presented. The obtained electron concentration and electron mobility vs. temperature and $\mathrm{Cr}$ concentration data are interpreted and discussed within the model assuming that $\mathrm{Cr}$ in $\mathrm{PbTe}$ forms a donor state resonant with the conduction band.

PACS numbers: $72.20 . \mathrm{Fr}, 72.10 . \mathrm{Fk}, 72.80 . \mathrm{JC}$

Contrary to the case of II-VI compounds - where donors with ground state energy that is resonant with the conduction band are not common (and only one, namely $\mathrm{Fe}$ in IIgSe [1], being well evidenced) - in IV-VI compounds a situation is different. In PbTe, for example, at least three impurities: $\mathrm{In}, \mathrm{Cr}$ and $\mathrm{Ti}$ are known as forming states resonant with conduction band [2].

The experimental evidences indicating that chromium atoms (substituting $\mathrm{Pb}$ ions in $\mathrm{PbTe}$ ) give rise to a resonant donor state superimposed on the conduction band continuum were obtained by means of transport (see [3] references therein) and magnetic [4] studies. The experimental data are consistent with the following picture: at the doping level $n_{\mathrm{Cr}}^{*}<1.3 \times 10^{19} \mathrm{~cm}^{-3}$ due to the self-ionization process $\left(\mathrm{Cr}^{2+} \Rightarrow \mathrm{Cr}^{3+}+e\right)$ chromium impurity exists in $\mathrm{Cr}^{3+}$ state and the conduction electron concentration is proportional to the amount of Cr. Above this characteristic chromium concentration $n_{\mathrm{Cr}}^{*}$, the conduction electron concentration becomes constant, independent of further doping (see Fig. 1 of Ref. [5]) since the Fermi level is then trapped at the $\mathrm{Cr}$ level and further self-ionization process is impossible. In this high doping regime only a part of $\mathrm{Cr}$ impurities are ionized. $\mathrm{Cr}$ ions coexist in two charge states: $\mathrm{Cr}^{2+}$ and $\mathrm{Cr}^{3+}$ making this system similar to the system of Fe donors in $\mathrm{HgSe}$ for $n_{\mathrm{Fe}}>4.5 \times 10^{18} \mathrm{~cm}^{-3}$.

In analogy to $\mathrm{HgSe}: \mathrm{Fe}$ one can expect that also in $\mathrm{PbTe}: \mathrm{Cr}$, the mutual Coulomb interaction between the charges localized on impurities will cause a spatial correlation of charges localized on donors. The correlation, in consequence,

*This work is supported in part by the Committee for Scientific Research under grant No. 204829101 and by U.S.-Poland Maria Curie-Sklodowska Joint Found II through grant No. PAN/NSF-92-113. 
will reflect itself in a reduction of the scattering rate due to Coulomb potentials of charged centers. In the present study $\mathrm{PbTe}$ samples, doped with $\mathrm{Cr}$ up to $n_{\mathrm{Cr}} \approx 1 \times 10^{20} \mathrm{~cm}^{-3}$, obtained by the modified Bridgman method were investigated. The chemical compositions of the samples were determined by means of energy dispersive $\mathrm{X}$-ray fluorescence analysis.

The conduction electron concentration $n_{e}$ and the electron mobility $\mu_{e}$ were measured in the temperature range $3.5-300 \mathrm{~K}$. Figure 1 shows the temperature

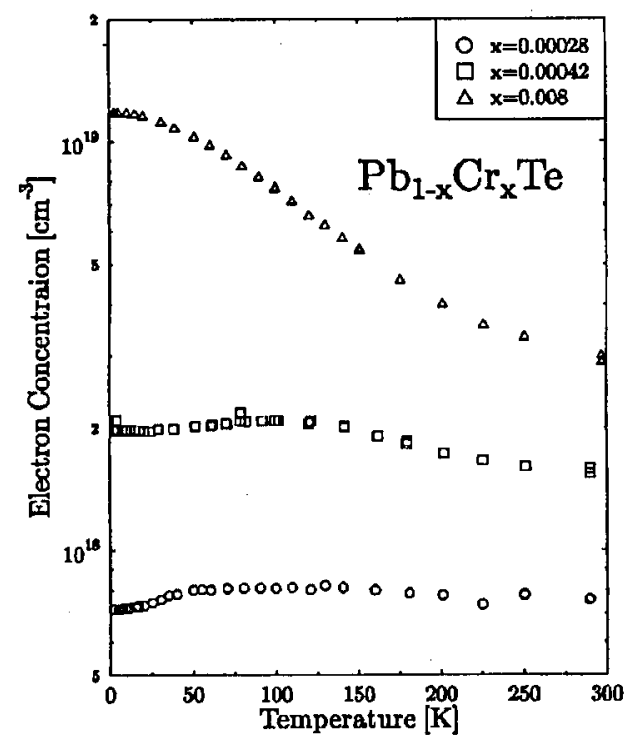

Fig. 1. The dependence of electron concentration on temperature for the three $\mathrm{Pb}_{1-x} \mathrm{Cr}_{x}$ Te samples.

dependence of the electron concentration for three samples doped with $\mathrm{Cr}$. Before measurements the samples were annealed in $\mathrm{Pb}$ vapour. The decrease in the electron concentration with temperature for the sample with $x=0.008$ may be explained assuming that the Fermi energy is pinned to the Cr state, whose energy. level shifts downwards with temperature relative to the conduction band edge. Thus the knowledge of the Fermi level energy determines the $\mathrm{Cr}^{2+}$ level energy at each temperature. In [3] and earlier papers the Fermi energy was calculated in the two-band approximation [6] and the position of the chromium level (i.e., the Fermi energy) for liquid He temperatures was found to be $E_{\mathrm{Cr}} \approx 100 \mathrm{meV}$ above the conduction band edge. This result is also found if the calculations are made with the help of the $\boldsymbol{k} \cdot \boldsymbol{p}$ model of Dimmock [7] with the set of band parameters determined by Hewes et al. [8]. The calculations based on parameters obtained from magnetooptical studies [9] lead to a different result $-E_{\mathrm{Cr}} \approx 70 \mathrm{meV}$.

The curve for the sample with $x=0.00042$ represents a different case. All chromium ions, at the lowest temperatures, are ionized to $\mathrm{Cr}^{3+}$ state in that sample. After an initial slight increase in the electron concentration with temperature 
(which, like in the case of the sample with the lowest electron concentration, may be attributed to the presence of defect states in the forbidden gap [10]) we observe a decrease in the electron concentration with temperature. This effect is due to the increasing, with temperature, probability of redistribution of the electrons between band states and the states localized on Cr. The lowest curve - corresponding to the sample molar fraction $x=0.00028$ of $\mathrm{Cr}$ as mentioned earlier exhibits similar behavior to that observed in $\mathrm{PbTe}[10]$.

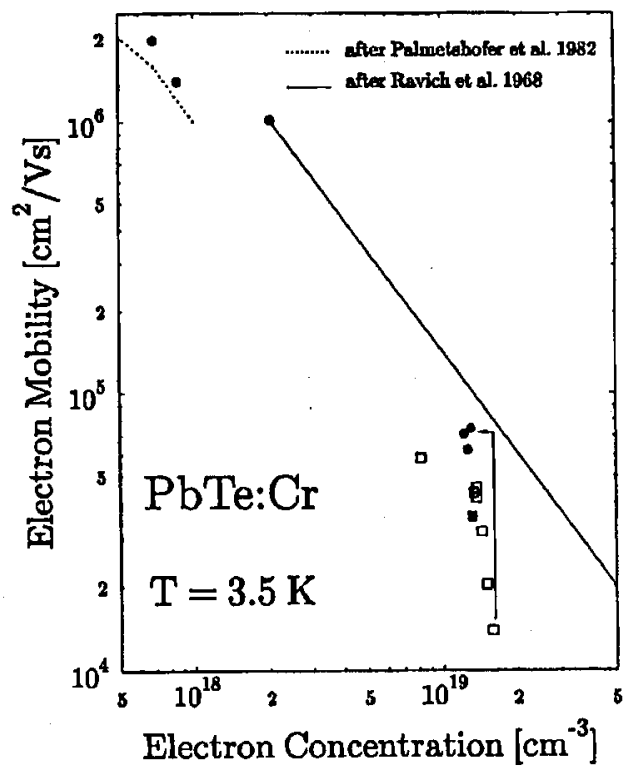

Fig. 2. The dependence of electron mobility on electron concentration in $\mathrm{PbTe:Cr}$ samples. The dotted line presents the results of the mobility calculation by Palmetshofer et al. [11], the solid curve presents the best known mobility values in PbTe, after Ref. [6]. The data obtained in this study are shown by various symbols. The annealed samples are presented as full circles, not annealed as open squares. The arrow connects the results for the sample with molar fraction $x=0.009$ of $\mathrm{Cr}$ obtained prior to annealing process and after annealing.

Figure 2 shows the dependence of electron mobilities on electron concentration. As a reference, the results of the mobility calculation by Palmetshofer et al. [11] and the curve representing the best known mobility values after Ref. [6] are also plotted. Our three samples with electron concentration lower then the critical one (i.e., $E_{\mathrm{Cr}} \approx 1.25 \times 10^{19} \mathrm{~cm}^{-3}$ ) display mobility values that are close to the best values reported in the literature on $\mathrm{PbTe}$. These samples were annealed in $\mathrm{Pb}$ or vacuum before measurements. The highest value of the mobility achieved at liquid He temperature in the present study was $2 \times 10^{6} \mathrm{~cm}^{2} /(\mathrm{V} \mathrm{s})$ for sample with $n=7 \times 10^{17} \mathrm{~cm}^{-3}$. It may be noted that the mobility value exceeds the theoretical prediction. This is due to the simplification of the calculations which 
underestimate the final mobility (see Addendum in [11]).

The electron mobility values for samples with electron concentrations corresponding to the mixed valence regime $\left(n \geq 1.3 \times 10^{19} \mathrm{~cm}^{-3}\right)$ are presented for both as-grown (open squares) and annealed (full circles) samples. Similarly to the case of the samples with smaller content of chromium, the best mobility values for our annealed samples are close to the best values observed in various investigations of the $\mathrm{PbTe}$ doped with nonresonant donors. The annealing process substantially improves the electron mobility, whereas its effect on electron concentration is minimal (see the line connecting two points corresponding to as-grown and annealed sample in Fig. 2). The fact that the electron concentration does not depend on annealing procedure supports additionally the thesis that the chromium impurity in $\mathrm{PbTe}$ forms a resonant donor state degenerated with the conduction band.

A question arises why the effect of the spatial correlation, so important in the case of $\mathrm{HgSe}: \mathrm{Fe}$, in the seemingly analogous system - $\mathrm{PbTe}: \mathrm{Cr}$ - does not influence the mobility values at low temperatures. The most likely answer is connected with the dielectric constant of $\mathrm{PbTe}$, which reaches very high values (1300 at $4 \mathrm{~K} \mathrm{[12]).} \mathrm{In} \mathrm{such} \mathrm{circumstances} \mathrm{the} \mathrm{scattering} \mathrm{on} \mathrm{charged} \mathrm{centers,} \mathrm{which}$ usually dominates at low temperatures, is ineffective and the electron mobility is limited by other scattering mechanisms. Therefore, the spatial correlation, even if present, does not affect greatly the observed mobility values. On the other hand the lack of the resonant scattering, a powerful mobility suppressing mechanism, indicates that, despite the high value of dielectric constant, the Coulomb gap forms in one electron density of states. The Coulomb gap, i.e., a depletion of the DOS at the Fermi level strongly suppresses the resonant scattering [1], which is otherwise clearly observed in $\mathrm{PbTe}$ doped with resonant impurities [2]. The formation of the Coulomb gap, in turn, is just another manifestation of the onset of Coulomb interaction-driven spatial correlation of impurity charges. Thus we take the absence of the resonant scattering in the samples studied here as an indirect indication of the existence of the spatially correlated system of charges localized on $\mathrm{Cr}$ ions in the mixed valence regime.

\section{References}

[1] J. Kossut, W. Dobrowolski, Z. Wilamowski, T. Dietl, K. Świątek, Semicond. Sci. Technol. 5, 260 (1990).

[2] V.I. Kaŭdanov, S.A. Nemov, Yu.I. Ravich, Sov. Phys. Semicond. 26, 113 (1992).

[3] L.M. Kashirskaya, L.I. Ryabova, O.I. Tananaeva, N.A. Shirokova, Sov. Phys. Semicond. 24, 848 (1990).

[4] T. Story, E. Grodzicka, B. Witkowska, J. Górecka, W. Dobrowolski, Acta Phys. Pol. A 82, 879 (1992).

[5] T. Story, Z. Wilamowski, E. Grodzicka, B. Witkowska, W. Dobrowolski, 22th Internat. School on Physics of Semiconducting Compounds, Jaszowiec 1993, to be published.

[6] Yu.I. Ravich, B.A. Efimova, I.A. Smirnov, The Methods of Investigations of Semiconductors in Application to Lead Chalcogenides, Nauka, Moscow 1968. 
[7] J.O. Dimmock, in: The Physics of Semimetals and Narrow Gap Semiconductors, Eds. L. Carter, R.T. Bate, Pergamon Press, New York 1971.

[8] C.R. Hewes, M.S. Adler, S.D. Senturia, Phys. Rev. B 7, 5195 (1973).

[9] G. Bauer, H. Pascher, W. Zawadzki, Solid State Commun. 7, 703 (1992).

[10] K. Lischka, Appl. Phys. A 29, 177 (1982).

[11] L. Palmetshofer, K.H. Gresslehner, L. Ratschbacher, A. Lopez-Otero, in: Lecture Notes in Physics, Eds. E. Gornik, H. Heinrich, L. Palmetshofer, Vol. 152, Springer Verlag, Berlin 1982, p. 391.

[12] H. Burkhard, G. Bauer, A. Lopez-Otero, Phys. Rev. B 18, 2935 (1978). 